\title{
Study on the Theory of Projectile Firing Process during Marching Fire
}

\author{
Liu Feifei \\ Institute of Launch Dynamics \\ Nanjing University of Science \& Technology \\ Nanjing, P. R. China \\ Liufeifei_paper@163.com \\ Yu Hailong \\ Institute of Launch Dynamics \\ Nanjing University of Science \& Technology \\ Nanjing, P. R. China \\ yhls02080888@sina.com
}

\author{
Rui Xiaoting * \\ Institute of Launch Dynamics \\ Nanjing University of Science \& Technology \\ Nanjing, P. R. China \\ ruixt@163.net \\ Zhang Jianshu \\ Institute of Launch Dynamics \\ Nanjing University of Science \& Technology \\ Nanjing, P. R. China \\ zhangdracpa@sina.com
}

\begin{abstract}
The initial disturbance of the projectile is the premier factor that takes effect on the marching fire accuracy of the conventional weapon, which depends on the projectile firing process of the conventional weapon. In this paper, the theory of projectile firing process of the conventional weapon during marching fire was studied. Combining the projetile motion equations in gun tube with the system dynamics equations which can be obtained by various multibody system dynamics methods, the motion of the projectile in barrel and the initial disturbance of the projectile can be exactly simulated. This work provides both theoretical foundation and simulation approaches for improving the marching fire accuracy of the conventional weapon.
\end{abstract}

Keywords-marching fire; projectile firing process; firing accuracy; initial disturbance

\section{INTRODUCTION}

The launch process has great influence on the dynamic property of weapons, especially on the marching fire accuracy of weapons. It is verified by the theory and tests that 1, 2, the initial disturbance of the projectile is the premier factor which takes effect on the marching fire accuracy of weapons. Meanwhile, the influence of road excitation, vibration and stabilization precision on the marching fire accuracy can also be reflected by the initial disturbance of the projectile. To a major extent, the marching fire accuracy of weapons depends on the behavior of its projectile firing process. The projectile firing process 3 is a novel subject which studies the principle of forces and the movements of the weapon system in the launch process. Further, it provides the theorem and techniques of control and principles of forces and movements for system design and test of dynamic properties of weapon systems. Developed for many years, theory of projectile firing process has come into the stage of engineering applications by Xiao-ting Rui, et al4-9. Fruitful achievement has been gained in improving the firing accuracy and reducing ammunition consumption. The launch dynamics of tanks and self-propelled artilleries marching fire were studied by many scholars10-12, however, the dynamics models of weapon systems are relatively simple, and the theory of projectile firing process during marching are imperfect. Thereby, it is impossible to get the accurate initial disturbance of the projectile, and thus difficult to discuss the way of improving the marching fire accuracy of weapons.

Aiming at these problems, this paper studied on the theory of projectile firing process of weapons during marching fire. Take a self-propelled and tank artillery as examples, the motion of the projectile in barrel, the initial disturbance of the projectile and the vertical target dispersion were exactly simulated, meanwhile, the results of simulation are verified by tests.

\section{THE PROJECTILE MOTION EQUATIONS IN GUN TUBE DURING MARCHING}

Based on the previous studies ${ }^{1-9,13}$, regarding the projectile as a rigid body, the elastic effect of projectile-barrel interaction modeled as the elastic contact forces between barrel and bourrelet, and between the barrel and the bearing band.

\section{A. The coordinate systems of the projectile motion in} gun tube

Based on literature [2], the barrel coordinate system $o_{4} x_{4} y_{4} z_{4}$ (b.c.s) is introduced. At the initial time, the origin $O_{4}$ of b.c.s and the origin $O_{3}$ of the artillery coordinate system $o_{3} x_{o}^{\prime} y_{o}^{\prime} z_{o}^{\prime}$ (a.c.s) match together, the positive direction of $x_{4}$-axis is along the tangent of barrel axis pointing to the muzzle, $y_{4}$-axis is perpendicular to $x_{4}$-axis in the vertical plane, $z_{4}$-axis is determined by the right-hand rule. The coordinate system is fixed with the barrel. The artillery coordinate system, the ground coordinate system (g.c.s), the projectile axis coordinate system (p.a.c.s) and the projectile coordinate system (p.c.s) are defined in ref. [2]. 
B. The differential equations of motion of the projectile mass center during marching fire

According to the Newton's second law of motion, the equations of motion of the projectile mass center (m.c.) during marching is

$$
\boldsymbol{F}_{\boldsymbol{C}}=m\left(\boldsymbol{a}_{\boldsymbol{C}}^{\prime}+\boldsymbol{A}_{4,0}^{\mathrm{T}} \boldsymbol{D}_{1} \ddot{x}_{p}+\ddot{\boldsymbol{r}}_{\boldsymbol{o}_{3} \boldsymbol{C}}\right) .
$$

Where,

$$
\begin{aligned}
& \boldsymbol{a}_{\boldsymbol{C}}^{\prime}=\boldsymbol{A}_{4,0}^{\mathrm{T}} \boldsymbol{A}_{1,4}^{\mathrm{T}} \ddot{\boldsymbol{r}}_{\boldsymbol{o}_{4}}+\boldsymbol{A}_{4,0}^{\mathrm{T}}\left(\boldsymbol{D}_{5}+2 \dot{x}_{p} \boldsymbol{D}_{\mathbf{4}}+\dot{x}_{p}^{2} \boldsymbol{D}_{\mathbf{2}}+2 \tilde{\boldsymbol{\omega}}_{4,1}\left(\boldsymbol{D}_{2}+\dot{x}_{p} \boldsymbol{D}_{\boldsymbol{I}}\right)+\dot{\tilde{\boldsymbol{\omega}}}_{4,1} \boldsymbol{r}_{\boldsymbol{o}_{4} \boldsymbol{o}_{3}}+\tilde{\boldsymbol{\omega}}_{4,1} \tilde{\boldsymbol{\omega}}_{4,1} \boldsymbol{r}_{\boldsymbol{o}_{4} \boldsymbol{o}_{3}}\right)+ \\
& \boldsymbol{A}_{\theta}^{\mathrm{T}}\left(\ddot{\boldsymbol{A}}_{\theta} \boldsymbol{r}_{\boldsymbol{o}_{3} \mathrm{C}}+2 \dot{\boldsymbol{A}}_{\theta} \dot{\boldsymbol{r}}_{\boldsymbol{o}_{3} \mathrm{C}}+2 \tilde{\boldsymbol{\omega}}_{\alpha}\left(\dot{\boldsymbol{A}}_{\theta} \boldsymbol{r}_{\boldsymbol{o}_{3} C}+\boldsymbol{A}_{\theta} \dot{\boldsymbol{r}}_{\boldsymbol{o}_{3} \mathrm{C}}\right)+\tilde{\boldsymbol{\omega}}_{\alpha} \tilde{\boldsymbol{\omega}}_{\alpha} \boldsymbol{A}_{\theta} \boldsymbol{r}_{\boldsymbol{o}_{3} \mathrm{C}}+\left(\boldsymbol{B}-\widetilde{\boldsymbol{\omega}}_{\psi}\left(\boldsymbol{A}_{\psi} \boldsymbol{\omega}_{4,1}\right)+\boldsymbol{A}_{\psi} \dot{\boldsymbol{\omega}}_{4,1}\right) \boldsymbol{A}_{\theta} \boldsymbol{r}_{\boldsymbol{o}_{3} C}\right) \\
& \boldsymbol{D}_{1}=\frac{\partial \boldsymbol{r}_{\boldsymbol{o}_{4} \boldsymbol{o}_{3}}}{\partial \boldsymbol{S}} ; \quad \boldsymbol{D}_{2}=\frac{\partial \boldsymbol{r}_{\boldsymbol{o}_{\boldsymbol{o}_{3}}}}{\partial \boldsymbol{t}} ; \quad \boldsymbol{D}_{4}=\frac{\partial^{2} \boldsymbol{r}_{\boldsymbol{o}_{4} \boldsymbol{O}_{3}}}{\partial \mathrm{s} \partial \boldsymbol{t}} ; \quad \boldsymbol{D}_{5}=\frac{\partial^{2} \boldsymbol{r}_{\boldsymbol{o}_{4} \boldsymbol{O}_{3}}}{\partial \boldsymbol{t}^{2}}
\end{aligned}
$$

the physical meanings of symbols and the calculation methods of parameters coincide with ref. [22,23].

\section{The differential equations of rotation of projectile during marching fire}

Taking the p.a.c.s as a moving coordinate system, the projectile geometrical center $o_{1}$ (g.c.) as basic point,

$$
\ddot{\gamma}\left[\begin{array}{c}
C \\
-(A-C) \beta_{D_{\eta}} \\
-(A-C) \beta_{D_{\xi}}
\end{array}\right]+\boldsymbol{J}_{o_{1}}\left[\begin{array}{c}
\delta_{2}^{I} \ddot{\delta}_{1}^{I} \\
-\ddot{\delta}_{2}^{I} \\
\ddot{\delta}_{1}^{I}
\end{array}\right]+m \widetilde{r}_{o_{1} C} \boldsymbol{A}_{2,0}\left(\boldsymbol{A}_{4,0}^{\mathrm{T}} \boldsymbol{D}_{1} \ddot{x}_{p}+\ddot{\boldsymbol{r}}_{o_{3} c}-\boldsymbol{B}_{5} \ddot{\gamma}\right)=\boldsymbol{M}_{\boldsymbol{o}_{1}}+\boldsymbol{M}_{\boldsymbol{I}} \cdot
$$

where

$$
\begin{aligned}
& \boldsymbol{M}_{\boldsymbol{1}}=-\boldsymbol{J}_{\boldsymbol{o}_{1}}\left(\boldsymbol{\Delta}+\tilde{\boldsymbol{\omega}}_{2,0} \boldsymbol{A}_{2,0} \boldsymbol{\omega}_{\theta}+\boldsymbol{A}_{2,0} \dot{\boldsymbol{\omega}}_{\theta}-\widetilde{\boldsymbol{\omega}}_{\delta} \boldsymbol{A}_{2,0} \boldsymbol{A}_{\theta}^{\mathrm{T}} \boldsymbol{\omega}_{\alpha}+\boldsymbol{A}_{2,0} \boldsymbol{A}_{\theta}^{\mathrm{T}}\left(\boldsymbol{B}+\tilde{\boldsymbol{\omega}}_{\psi} \boldsymbol{A}_{\psi} \boldsymbol{\omega}_{4,1}+\boldsymbol{A}_{\psi} \dot{\boldsymbol{\omega}}_{4,1}\right)\right) \\
& -\tilde{\boldsymbol{\omega}}_{2,1} \boldsymbol{G}_{\boldsymbol{o}_{1}}^{r}+\tilde{\boldsymbol{\omega}}_{2,1} \boldsymbol{J}_{\boldsymbol{o}_{1}} \boldsymbol{\omega}_{2,1}-\boldsymbol{m} \tilde{\boldsymbol{r}}_{\boldsymbol{o}_{1} \boldsymbol{C}} \boldsymbol{A}_{2,0}\left(\boldsymbol{a}_{\boldsymbol{C}}^{\prime}+\boldsymbol{A}_{\theta}^{\mathrm{T}}\left(\ddot{\boldsymbol{A}}_{\theta} \boldsymbol{B}_{3}+2 \dot{\boldsymbol{A}}_{\theta} \boldsymbol{B}_{\mathbf{4}}+2 \tilde{\boldsymbol{\omega}}_{\alpha}\left(\dot{\boldsymbol{A}}_{\theta} \boldsymbol{B}_{3}+\boldsymbol{A}_{\theta} \boldsymbol{B}_{\mathbf{4}}\right)+\right.\right. \\
& \left.\left.\left(\boldsymbol{B}-\tilde{\boldsymbol{\omega}}_{\psi} \boldsymbol{A}_{\psi} \boldsymbol{\omega}_{4,1}+\boldsymbol{A}_{\psi} \dot{\boldsymbol{\omega}}_{4,1}\right) \boldsymbol{A}_{\theta} \boldsymbol{B}_{\mathbf{3}}+\tilde{\boldsymbol{\omega}}_{\alpha} \tilde{\boldsymbol{\omega}}_{\alpha} \boldsymbol{A}_{\theta} \boldsymbol{B}_{\mathbf{3}}\right)-\dot{\gamma}^{2} \boldsymbol{B}_{3}\right)-\dot{\gamma}^{2} \boldsymbol{N}+\frac{\tilde{\mathrm{d}} \boldsymbol{J}_{\boldsymbol{o}_{1}}}{\mathrm{~d} t} \boldsymbol{\omega}_{2,1}+\tilde{\boldsymbol{\omega}}_{2,1} \boldsymbol{J}_{\boldsymbol{o}_{1}} \boldsymbol{\omega}_{2,1}
\end{aligned}
$$

the physical meanings of symbols and the calculation methods of parameters coincide with ref. [22,23].

\section{THE SYSTEM DYNAMICS EQUATIONS OF WEAPONS}

Multibody system dynamics provides a strong tool for dynamic performance estimation and optimizing design of weapon ${ }^{14}$. The present multibody system dynamics methods mainly include Newton-Euler vector mechanical method, Jourdam principle, Lagrange method, Gauss principle, Transfer matrix method of multibody system, and so on ${ }^{15-19}$.

Taking a self-propelled artillery and tank as examples, applying the transfer matrix method for multibody system (MSTMM) and the automatic deduction theorem of overall transfer equation ${ }^{20,21}$, the system dynamics equations of the self-propelled artillery and tank were derived in ref. $[22,23]$. The topological graph of the selfpropelled artillery dynamics model is shown in Fig .1. more details about the model can refer to literature [22,23]. 


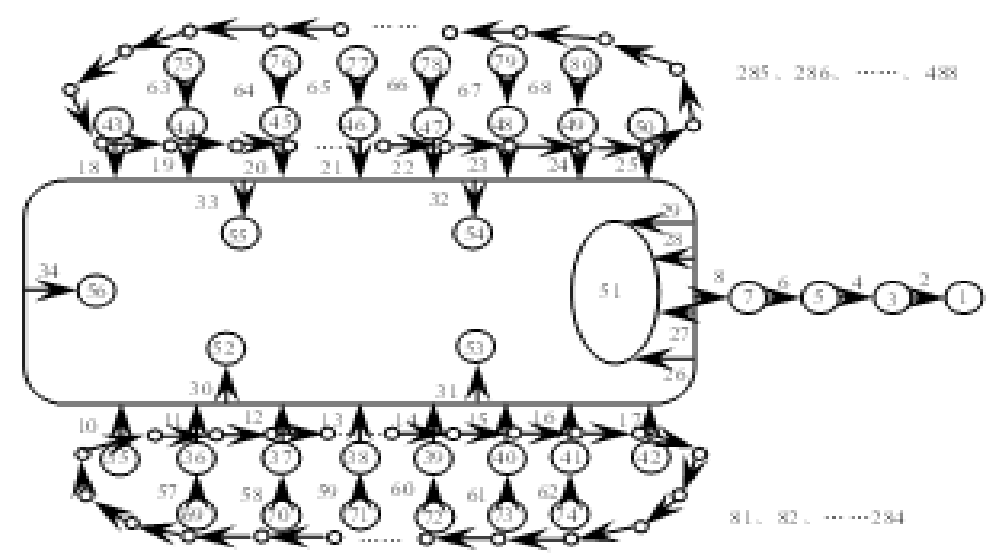

Figure 1. Topological graph of launch dynamics of a self-propelled artillery.

\section{THE SIMULATION OF THE SELF-PROPELLED ARTILLERY AND TANK MARCHING FIRE AND ITS TEST VERIFICATION}

On the base of the preceding projectile firing process equations and the multibody dynamic equations of the self-propelled artillery and tank during marching, the simulation program of the launch dynamics of the selfpropelled artillery and tank were compiled, and the simulation results of the self-propelled artillery and tank during marching fire and fire at rest were given in Fig .2 Fig .6 and Table I - Table II.

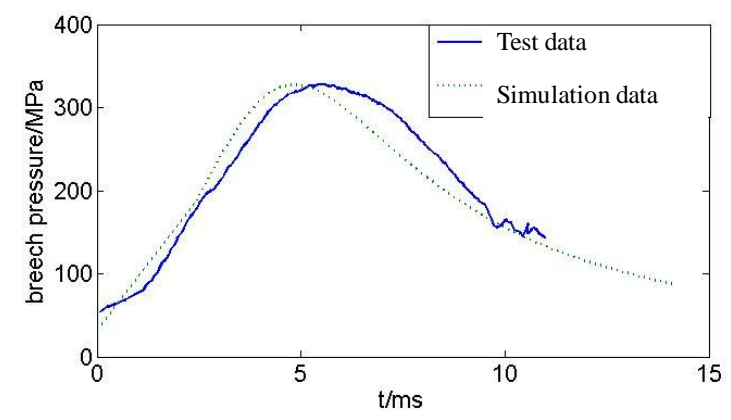

Figure 2. The results of the breech pressure obtained by computation and test.

Fig .2 is the curves of the breech pressure obtained by computation and test, the simulation error of the maximum breech pressure is $1.13 \%$ and the simulation curves of the breech pressure are well fit with experimental results.

Fig .3 - Fig .4 are the simulation curves of impact force of the self-propelled artillery between the bourrelet and the barrel, the projectile vertical swing angular velocity of the self-propelled artillery respectively. Marching fire conditions: the self-propelled artillery speed is $20 \mathrm{Km} / \mathrm{h}$, road profile is level $\mathrm{D}$, fire angle is $51^{\circ}$.
Computational results of the impact force of the selfpropelled artillery

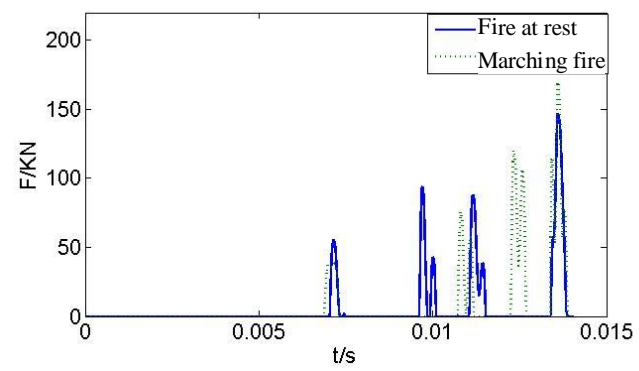

Figure 3. between the barrel and the bourrelet.

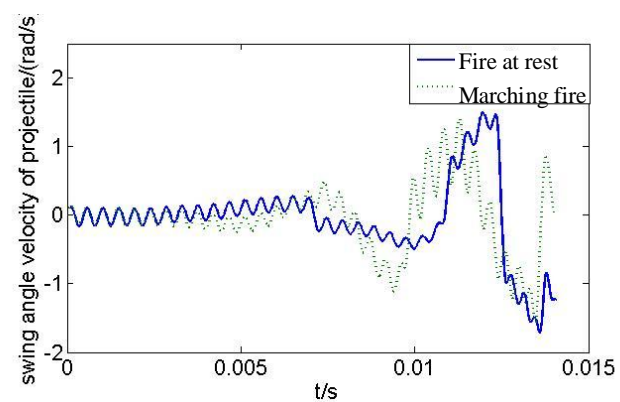

Figure 4. Computational results of the projectile vertical swinging angular velocity of the self-propelled artillery.

Fig $.5-$ Fig .6 are the simulation curves of impact force of the tank between the bourrelet and the barrel, the projectile vertical swing angular velocity of the tank respectively. Marching fire conditions: the tank speed is $40 \mathrm{Km} / \mathrm{h}$, road profile is level $\mathrm{D}$, fire angle is $0^{\circ}$.

The barrel-projectile impact force and vertical swing angular velocity of projectile during marching fire are significantly distinguished from those during fire at rest. 


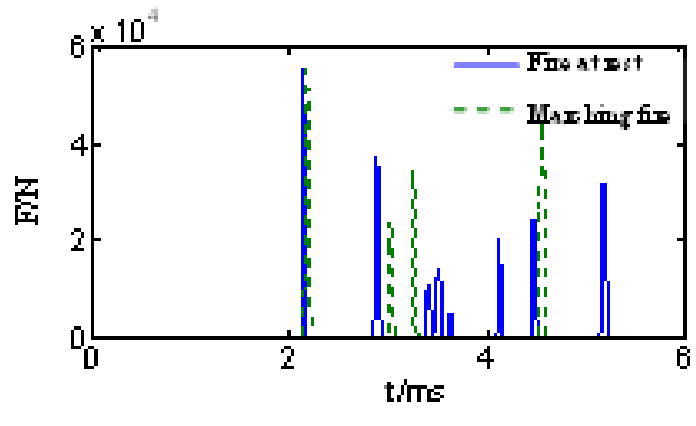

Figure 5. Computational results of the impact force of the tank between the barrel and the bourrelet.

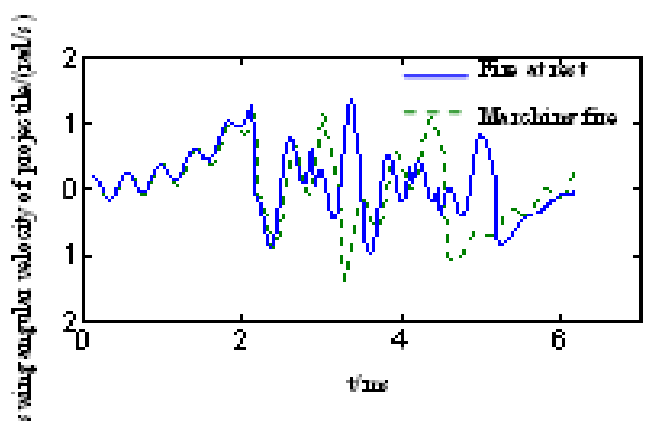

Figure 6. Computational results of the projectile vertical swinging angular velocity of the tank.

Table I is the simulation results of initial disturbance of the self-propelled artillery and Table II is the results of fire dispersion of the self-propelled artillery obtained by computation and test, the simulation errors during fire at rest are $8.7 \%$ (distance dispersion) and $7.2 \%$ (direction dispersion). And the results of fire dispersion obtained by computation during marching fire are worse than those during fire at rest, the situation is suitable in real cases.

TABLE I. THE RESULTS OF INITIAL DISTURBANCE OF THE PROJECTILE OBTAINED BY COMPUTATION

\begin{tabular}{|c|c|c|c|c|}
\hline$y_{O_{3} c} /(\mathbf{m m})$ & $z_{O_{3} c} /(\mathbf{m m})$ & $\gamma /(\mathbf{m r a d})$ & $\delta_{1}^{I} /(\mathbf{m r a d})$ & $\delta_{2}^{I} /(\mathbf{m r a d})$ \\
\hline-0.200 & -0.031 & 9.302 & -0.769 & -0.103 \\
\hline$\dot{y}_{o_{3} c} /(\mathbf{m m})$ & $\dot{z}_{O_{3} c} /(\mathbf{m m})$ & $\dot{\gamma} /(\mathbf{m r a d})$ & $\dot{\delta}_{1}^{I} /(\mathbf{m r a d})$ & $\dot{\delta}_{2}^{I} /(\mathbf{m r a d})$ \\
\hline 0.072 & -0.395 & 1621.4 & -0.025 & -1.03 \\
\hline
\end{tabular}

TABLE II. THE RESULT OF FIRE DISPERSION OBTAINED BY COMPUTATION AND TEST

\begin{tabular}{|l|l|c|}
\hline \multirow{2}{*}{ Simulation and test result } & \multicolumn{2}{|c|}{ Fire dispersion/m } \\
\cline { 2 - 3 } & Distance dispersion & Direction dispersion \\
\hline Simulation result (at rest) & 161.3 & 51.2 \\
\hline $\begin{array}{l}\text { Simulation result } \\
\text { (on the march) }\end{array}$ & 184.2 & 53.4 \\
\hline Test result (at rest) & 176.7 & 48.7 \\
\hline
\end{tabular}

The above results have shown that the projectile firing process equations during marching established and the simulation program of the self-propelled artillery and tank launch dynamic compiled in this paper are correct and feasible.

\section{CONCLUSIONS}

The projectile motion equations in gun tube during marching fire were deduced. Take a self-propelled artillery and tank as examples, combing the projectile motion equations during marching fire with the multibody system dynamics equations of the self-propelled artillery and tank obtained by applying the MSTMM, a program of the launch dynamics of the self-propelled artillery and tank was compiled. Via the program, the motion of the projectile in the barrel, initial disturbance of the projectile and the fire dispersion are simulated.

The simulation curves of the breech pressure of the self-propelled artillery are well fit with experimental results, the simulation error of the maximum breech pressure of the self-propelled artillery is $1.13 \%$ and the simulation errors of the self-propelled artillery fire dispersion during fire at rest are $8.7 \%$ (distance dispersion) and $7.2 \%$ (direction dispersion). The simulation results show that the fire dispersion during marching fire are worse than those during fire at rest. This work provides both theoretical foundation and simulation approaches for improving the marching fire accuracy of the conventional weapon.

\section{ACKNOWLEDGMENT}

This work was financially supported by the Equipment Pre-research Mutual Application Techniques Foundation of China (Grant No.9140A10041013BQ02143) and the National Natural Science Foundation of China (Grant no.61304137).

\section{REFERENCES}

[1] RUI Xiao-ting, LIU Yi-xin, YU Hai-long. Launch dynamics of tank and self-propelled artillery [M]. Beijing: Science Press, 2011.

[2] RUI Xiao-ting, YANG Qi-ren. Launching process theory [M] Nanjing: Southeast University Press, 1992.

[3] YUN Lai-feng. Study on launch dynamics of self-propelled artillery [D]. Nanjing: Nanjing University of Science and Technology, 2002.

[4] RUI Xiao-ting, YUN Lai-feng, et al. A study on the launch dynamics of multiple launch rocket system $[\mathrm{J}]$. Acta armamentarii, 2004, 25(5): 556-561.

[5] WANG Guo-ping, RUI Xiao-ting, CHEN Wei-dong. Dispersion Simulation Technique of Multiple Tube Weapon [J]. Journal of simulation, 2004, 16(5): 963-966.

[6] WANG Guo-ping, RUI Xiao-ting. Study on dynamics of long range multiple launch rocket system $[\mathrm{J}]$. Journal of dynamics and control, 2004,2(1): 59-64.

[7] RUI Xiao-ting. Launch dynamics of multibody system and its applications [J]. Engineering Sciences, 2011, 13(11): 76-82.

[8] WANG Guo-ping, RUI Xiao-ting, et al. Dynamics analysisfor wheeled and tracked multiple launch rocket system [J]. Acta armamentarii, 2012, 33(11): 1286-1290.

[9] http://dx.doi.org/10.1155/2014/308049 [Accessed on 10 September 2014] [J/OL].

[10] FENG Chang-gen, WEN Bo, et al. Dynamic analysis of a selfpropelled gun firing on the move [J]. Acta armamentarii, 2002, 23(4): 457-461.

[11] MIN Jian-ping, TAN Jun-jie, et al. Study on dynamics of selfpropelled guns at firing on the move $[\mathrm{J}]$. Journal of vibration and shock, 2003, 22(4): 88-90.

[12] LI Jian-feng, WANG Jian, et al. Research on random response for tracked vehicle marching fire [J]. Vehicle and power technology, 2009,3: 9-12.

[13] MARTING T S, ROBERT S B. Projectile motion in a flexible gun tube [R]. ADA140737, 1984. 
[14] Rong Bao. Developments of studies on multibody system dynamics[J]. Journal of vibration and shock, 2011, 30(7), 178187.

[15] Wittenburg J. Dynamics of multibody systems[M]. Springer, New York, 2008.

[16] Roberson R E, Schwertassek R. Dynamics of multibody systems[M]. Springer-Verlag, Berlin, 1988.

[17] Shabana A A. Dynamics of multibody systems[M]. Cambridge university press, 2013.

[18] De Jalón G J, Bayo E. Kinematic and dynamics of mutibody system: The real time challenge[M]. New York: Springer-Verlag. 1994.
[19] Huston. Multibody dynamics[M]. Boston: ButterworthHeinemann, 1990

[20] RUI Xiao-ting, YUN Lai-feng, LU Yu-qi, et al. Transfer matrix method of multi-body system and its applications [M]. Beijing: Science Press, 2008.

[21] http://dx.doi.org/10.1155/2014/378047 [Accessed on 10 September 2014] [J/OL].

[22] LIU Feifei, RUI Xiaoting, et al. Study on launch dynamics of the self-propelled artillery marching fire [J]. Journal of vibration Engineering, in press.

[23] LIU Feifei, RUI Xiaoting, et al. Study on the influence of barrels' flexibility on the launch dynamics of the tank during marching fire $[\mathrm{J}]$. Journal of vibration and shock, in press. 